\title{
ENSAIO MECÂNICO DE TENSÃO-DEFORMAÇÃO SOB TRAÇÃO: UM ESTUDO INTEGRADO COM A DISCIPLINA DE CÁLCULO
}

\author{
Cyro Martins Passos de Azevedo \\ Graduando de Engenharia Mecânica/ ISECENSA \\ cyro_martins_azevedo@hotmail.com \\ Laio Hentzy Godinho \\ Graduando de Engenharia Mecânica /ISECENSA \\ laiohg@hotmail.com
}

\author{
Maria Aparecida Laterça Saraiva \\ Graduanda de Engenharia Mecânica/ ISECENSA \\ marialatercas@gmail.com
}

\section{Silas das Dores Alvarenga}

Especialista em Sistemas Offshore/ISECENSA

salvarenga@iff.edu.br

\author{
Márcia Valéria Azevedo de Almeida Ribeiro \\ Mestre em Educação Matemática/ISECENSA \\ mvaleria@censanet.com.br
}

\section{RESUMO}

É importante que o Engenheiro conheça propriedades mecânicas fundamentais dos materiais que serão empregados na execução de projetos. Por meio de ensaios mecânicos, é possível determinar propriedades sobre o material a ser empregado em projetos específicos. Um dos ensaios mecânicos mais simples e mais utilizado é o ensaio de tração que permite conhecer como um material reage quando submetido a esforços de tração. Os Ensaios de Tração fornecem dados quantitativos que permitirão cálculos matemáticos para a determinação de parâmetros importantes de modo que o Engenheiro possa decidir quanto à utilização de um material específico para determinado fim. Sendo assim, esse trabalho tem como objetivo promover o estudo integrado de Ensaio Mecânico de tensão-deformação sob tração com a disciplina de Cálculo. Para alcançar o objetivo proposto, foi feita uma revisão bibliográfica e para a realização dos ensaios de tração utilizou-se a Máquina Universal de Ensaios. Considera-se que esse trabalho dá significado ao estudo da disciplina de Cálculo na Engenharia e faz com que os alunos tenham um olhar diferenciado para o estudo das disciplinas técnicas, percebendo o quanto a Matemática é importante no seu curso de Engenharia e que esta fará a diferença quando estiverem em suas atividades profissionais como Engenheiros.

Palavras-chave: Ensaio de Tração; Tensão; Deformação; Cálculo. 


\title{
CENTRO EDUCACIONAL NOSSA SENHORA AUXILIADORA \\ INSTITUTOS SUPERIORES DE ENSINO DO CENSA \\ CENTRO DE PESQUISA E PÓS-GRADUAÇÃO
}

\begin{abstract}
It is important that the engineer knows the fundamental mechanical properties of the materials that will be used in the execution of the projects. Through the use of the mechanical tests it is possible to determine the properties of the material that will be used in specific project. One of the simpler and most common mechanical tests is the traction test, which will allow us to know how a material will react under traction effort. The traction tests provides quantitative data that will allow mathematical calculation for determining of important parameters so that the engineer can decide on the utilization of a specific material to a determinate end. Therefore, this project has as objective to promote the integrated study of Mechanical Tension - Deformation Test with the calculus discipline. To achieve the proposed objective, a bibliographic review was done, and for the realization of the traction test, the Universal test Machine was used. It is considered that this work gives meaning to the study of the calculus discipline in their engineering course and make that the students have a differential look to the study of the technical disciplines, realizing how much math is important in his engineering course and that it will make the difference when they are in their professional activities as engineers.
\end{abstract}

Keywords: Traction test; Tension; Deformation; Calculus.

\section{INTRODUÇÃO}

A mecanização dos sistemas de produção teve início na Europa, no século XVIII, com a Revolução Industrial. Esta nos trouxe algumas inovações tais como o emprego do aço, a utilização de combustíveis derivados do petróleo e a invenção do motor à explosão.

O desenvolvimento da indústria vem exigindo a utilização de materiais adequados para a fabricação de determinados componentes, de modo a atender a uma demanda cada vez mais exigente e competitiva.

Ao desenvolver um projeto de Engenharia, é de grande importância que o engenheiro tenha conhecimento de propriedades mecânicas fundamentais dos materiais que serão empregados na execução do projeto.

Callister e Rethwisch (2013) ressaltam que um dos ensaios mecânicos de tensão-deformação mais comuns é conduzido sob tração e, por isso, é denominado ensaio de tração o qual é empregado para caracterizar várias propriedades mecânicas dos materiais.

Lopes (2011) destaca que os ensaios mecânicos permitem a determinação de propriedades mecânicas que se referem ao comportamento do material quando submetido à ação de esforços, e que são expressas em função de tensões e/ou deformações.

Segundo Lopes (2011), o ensaio de tração consiste na aplicação gradativa de uma carga de tração uniaxial nas extremidades de um corpo de prova. Ressalta ainda que:

o ensaio de tração pode ser utilizado para avaliar diversas propriedades mecânicas dos materiais de grande importância em projetos de máquinas e equipamentos mecânicos; é também bastante utilizado como teste para controle de especificações da matéria prima fornecida. Sua ampla utilização na indústria de componentes mecânicos deve-se à vantagem de fornecer dados quantitativos das características mecânicas dos materiais, como limite de resistência à tração, limite de escoamento, módulo de elasticidade, módulo de resiliência, módulo de tenacidade, ductilidade, coeficiente de encruamento e coeficiente de resistência (LOPES, 2011, p.130).

Persp. Online: exatas \& eng., Campos dos Goytacazes, 15 (06) 29 - 35 - 2016 


\section{CENTRO EDUCACIONAL NOSSA SENHORA AUXILIADORA \\ INSTITUTOS SUPERIORES DE ENSINO DO CENSA \\ CENTRO DE PESQUISA E PÓS-GRADUAÇÃO}

Para a realização dos ensaios de tração, utilizou-se a Máquina Universal de Ensaios, que se encontra no Laboratório de Ensaios Destrutivos do ISECENSA (Institutos Superiores de Ensino do CENSA).

Os Ensaios de Tração fornecem dados quantitativos que permitem cálculos matemáticos para a determinação de parâmetros e coeficientes importantes, citados anteriormente por Lopes (2011), para a análise de materiais.

O Ensaio de Tração é usado para caracterizar o material na sua própria fabricação, no controle de qualidade, no recebimento de material, na pesquisa de novos materiais, na identificação/caracterização de um material desconhecido e na investigação de falhas mecânicas.

Por meio do Ensaio Mecânico sob Tração foi obtido um diagrama de tensão-deformação, muito útil para complementar a análise matemática envolvida no processo. Segundo Hibbeler (2010), esse diagrama é bastante importante na Engenharia porque proporciona os meios para se obterem dados sobre resistência à tração de um material.

A motivação para desenvolver este trabalho reside no fato de mostrar para o aluno a necessidade crescente de aplicar conhecimentos matemáticos estudados na disciplina de Cálculo no decorrer do curso de Engenharia; proporcionando, assim, uma relação dinâmica entre teoria e prática, culminando numa integração disciplinar.

Campos (2009) nos chama a atenção que:

As aulas teóricas-práticas são mais agradáveis e o aluno desperta mais para o assunto que está sendo ministrado. Além disso, participa mais da aula, por meio de dúvidas. Aplicação do Cálculo a outras áreas faz com que o aluno se sinta mais a vontade, as dúvidas "onde vou aplicar esse conhecimento?" desaparecem! (Campos, 2009, p.2)

Diante do exposto, este trabalho se destina a responder à seguinte questão de pesquisa: "Como integrar os estudos adquiridos em Cálculo ao ensino do Ensaio Mecânico de Tensão-Deformação sob tração?". Sendo assim, tem como objetivo geral promover o estudo integrado de Ensaio Mecânico de tensãodeformação sob tração com a disciplina de Cálculo.

Os objetivos específicos foram definidos da seguinte forma: (i) estudar os fundamentos teóricos relativos ao Ensaio Mecânico de tração e as fórmulas matemáticas envolvidas; (ii) operar a máquina Universal de Ensaios Mecânicos; (iii) identificar as deformações elástica e plástica; (iv) analisar o gráfico de Tensão-Deformação; (v) calcular parâmetros importantes tais como o Módulo de Elasticidade, Módulo de Resiliência e Módulo de Tenacidade.

Este trabalho se justifica pelo fato de ter um caráter integrador e interdisciplinar; buscando, assim, a formação integral do engenheiro.

Segundo Tomaz e David (2013), a interdisciplinaridade pode ser esboçada por meio de diferentes propostas, com diferentes concepções. Entre elas, aquelas que defendem um ensino aberto para inter-relações entre a Matemática e outras áreas do saber científico ou tecnológico. 


\section{CENTRO EDUCACIONAL NOSSA SENHORA AUXILIADORA \\ INSTITUTOS SUPERIORES DE ENSINO DO CENSA \\ CENTRO DE PESQUISA E PÓS-GRADUAÇÃO}

\section{METODOLOGIA}

Inicialmente, foi realizada uma pesquisa bibliográfica em livros, artigos, e textos na internet, com o objetivo de fundamentar o trabalho e aprofundar os estudos do tema em questão.

Para fazer os ensaios de tração, os alunos utilizaram a Máquina Universal de Ensaios Mecânicos, EMIC DL-2000, capacidade máxima de 20kN (Figura 1), existente no Laboratório de Ensaios Destrutivos do ISECENSA.

Durante o ensaio de tração, a máquina fornece um relatório com dados relevantes e um gráfico de tensão-deformação. A partir dos dados obtidos neste relatório e da análise do gráfico, foi possível observar as fases elástica e plástica e efetuar cálculos de parâmetros quantitativos, como Módulo de Elasticidade ou Módulo de Young, Módulo de Resiliência e Módulo de Tenacidade.

Nos ensaios de tração, foram utilizados corpos de prova de aço carbono 1020, com a forma de um cilindro circular reto, com aproximadamente $4 \mathrm{~mm}$ de diâmetro e comprimento que variava de $230 \mathrm{~mm}$ a 250mm (Figura 2).

Semanalmente, os membros da equipe de pesquisa se reuniam para tratar de questões relacionadas ao trabalho, tais como: estudo do tema, realização dos ensaios de tração no laboratório, análise dos gráficos obtidos nos ensaios e realização de cálculos.

\section{DESENVOLVIMENTO}

Com a finalidade de realizar a atividade integrada e atingir os objetivos propostos neste trabalho, reuniram-se num projeto de iniciação científica do ISECENSA, intitulado PROVIC (Programa Voluntário de Iniciação Científica), uma professora de Cálculo, coordenadora desse trabalho, um professor da área técnica e três alunos de Engenharia Mecânica do ISECENSA.

Feitos os estudos iniciais sobre o tema a ser trabalhado, marcamos um encontro no Laboratório de Ensaios Destrutivos. Nesse dia, sob a orientação do professor da área técnica, responsável pelo laboratório, os alunos fizeram um ensaio de tração em um corpo de prova, usando a Máquina Universal de Ensaios Mecânicos (Figura 1). Todo o trabalho de tração feito pela máquina era registrado em um gráfico. Os gráficos obtidos nos ensaios recebem o nome de diagrama de tensão-deformação (Figura 3).

Os alunos se mostraram motivados e acompanharam todo o processo, prestando atenção às explicações dos professores. Fizeram perguntas e tiraram algumas dúvidas que surgiram durante o ensaio. Após esse ensaio inicial, os alunos voltaram outras vezes ao laboratório e realizaram outros ensaios.

Com os estudos desenvolvidos, foi possível identificar no gráfico as fases elástica e plástica, o ponto $\mathrm{P}$ que representa o limite elástico e o ponto R onde acontece a ruptura. 


\section{CENTRO EDUCACIONAL NOSSA SENHORA AUXILIADORA \\ INSTITUTOS SUPERIORES DE ENSINO DO CENSA \\ CENTRO DE PESQUISA E PÓS-GRADUAÇÃO}

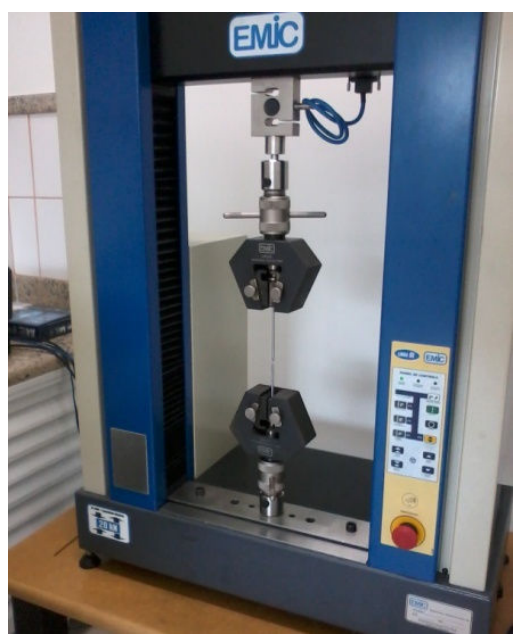

Figura 1 - Máquina Universal de Ensaios (ISECENSA).

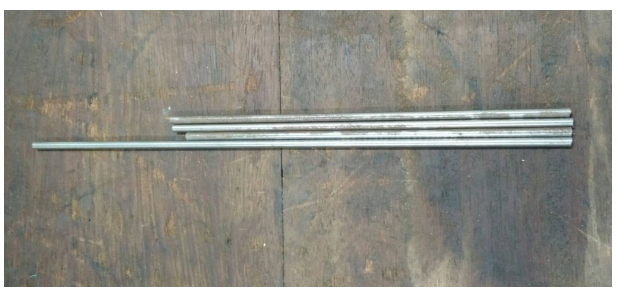

Figura 2 - Corpos de prova.

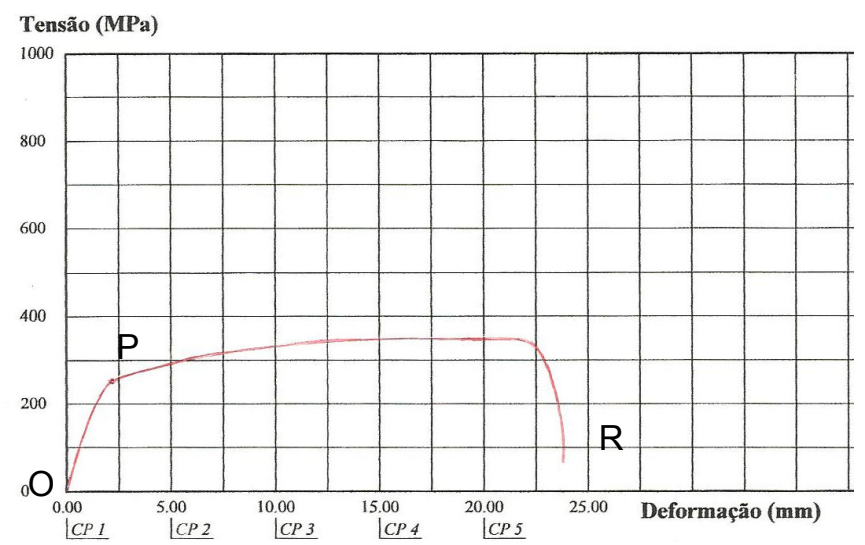

Figura 3 - Diagrama tensão-deformação.

$\mathrm{O}$ intervalo que contém o segmento de reta $\mathrm{OP}$, no gráfico, representa a fase de comportamento elástico. Na fase elástica, as deformações $(\varepsilon)$ são diretamente proporcionais às tensões $(\sigma)$ aplicadas (Lei de Hooke), por isso o comportamento linear nessa região, expresso matematicamente por $\sigma=\mathrm{E} . \varepsilon$, sendo $\mathrm{E}$ a constante de proporcionalidade, denominada módulo de elasticidade ou módulo de Young. Lopes (2011) destaca que a elasticidade é a capacidade que o material tem de se deformar quando submetido a um esforço e de voltar à forma original quando o esforço cessa.

Segundo Callister e Rethwisch (2013), o processo de deformação no qual a tensão e a deformação são proporcionais é chamado de deformação elástica e o gráfico da tensão (ordenada) em função da deformação (abscissa) é um segmento linear, cuja inclinação corresponde ao módulo da elasticidade.

Os alunos calcularam o módulo da elasticidade (E), ou seja, o coeficiente angular do segmento de reta na região elástica. Marcaram no gráfico alguns pontos na região elástica e dividiram o valor da tensão pelo valor da deformação $\left(E=\frac{\sigma}{\varepsilon}\right)$. Observaram resultados aproximadamente iguais, ou seja, estavam calculando o módulo da elasticidade. Segundo Lopes (2011), a inclinação (coeficiente angular) do segmento linear corresponde ao módulo de elasticidade do material, e é a medida de rigidez ou de resistência do 


\section{CENTRO EDUCACIONAL NOSSA SENHORA AUXILIADORA \\ INSTITUTOS SUPERIORES DE ENSINO DO CENSA CENTRO DE PESQUISA E PÓS-GRADUAÇÃO}

material à deformação elástica; ou seja, quanto maior esse módulo, menor será a deformação elástica resultante da aplicação de uma determinada tensão (maior rigidez). Essa propriedade é muito importante na escolha de materiais destinados à fabricação de molas.

É importante destacar que, para a realização dos cálculos, os alunos, inicialmente, dividiram cada valor do alongamento $(\Delta \ell)$ apresentado no eixo horizontal do gráfico mostrado na figura 3 pelo comprimento $(\ell)$ do corpo de prova, em milímetros; obtendo, assim, realmente a deformação $\left(\varepsilon=\frac{\Delta \ell}{\ell}\right)$. O que aparece no eixo horizontal do gráfico apresentado na figura 3 como "deformação" é na verdade o alongamento, em milímetros, que o corpo de prova está sofrendo no momento do ensaio. Sendo assim, a deformação é adimensional ( $\mathrm{mm} / \mathrm{mm})$.

Terminada a fase elástica, tem-se início a fase plástica, em que ocorre a deformação permanente do material, mesmo que cesse a tensão. Segundo Lopes (2011), a plasticidade é a capacidade que o material tem de se deformar quando submetido a um esforço, e de manter essa forma quando o esforço desaparece. Ele, ainda, destaca que essa propriedade é importante em processos de fabricação os quais exigem conformação mecânica, tais como: prensagem, laminação, fabricação de chapas e tubos. O ponto $\mathrm{R}$ marcado no gráfico corresponde à ruptura do material.

Voltando à região elástica, foi possível calcular o Módulo de Resiliência, que corresponde à área do triângulo na região elástica, ou seja, é a área entre o segmento OP e o eixo horizontal. Beer et al.,(2015) destacam que a capacidade de uma estrutura para resistir a uma força de impacto sem se deformar permanentemente depende claramente da resiliência do material utilizado. Hibbeler (2010) nos diz que, em termos físicos, a resiliência de um material representa sua capacidade de absorver energia sem sofrer qualquer dano permanente.

Os alunos fizeram o cálculo do Módulo de Resiliência com facilidade, pois tinham apenas de encontrar a área do triângulo. Alguns autores, também, indicam que essa área pode ser calculada por uma integral definida, fato entendido por eles, pois estudaram na disciplina de Cálculo como calcular áreas de regiões sob uma curva por meio de integrais definidas.

Outro importante parâmetro calculado foi o Módulo de Tenacidade que corresponde à área inteira sob a curva tensão-deformação até o ponto de ruptura R (figura 3). Beer et al.,(2015) destacam que a área abaixo de toda a curva da tensão em função da deformação foi definida como o módulo de tenacidade e é uma medida de energia total que pode ser absorvida pelo material.

Segundo Hibbeler (2010), essa propriedade é importante no projeto de elementos estruturais que possam ser sobrecarregados acidentalmente. Destaca, ainda, que materiais com alto módulo de tenacidade sofrerão grande distorção devido à sobrecarga; contudo, podem ser preferíveis aos que têm baixo valor de módulo de tenacidade, pois esses podem sofrer ruptura repentina sem dar nenhum sinal dessa ruptura iminente.

Nas pesquisas feitas, foi possível observar que os autores, de modo geral, não mostram como efetuar o cálculo do Módulo de Tenacidade. Alguns apresentam fórmulas que irão fornecer valores aproximados desse parâmetro.

Neste trabalho, foi realizado o cálculo do Módulo de Tenacidade, usando métodos numéricos estudados na disciplina de Cálculo Numérico. Conforme já definido, o Módulo de Tenacidade corresponde à área inteira sob a curva tensão-deformação até o ponto de ruptura. Como se desconhecia a função, a área da região pôde ser aproximada por Integração Numérica, utilizando a Regra do Trapézio, a partir dos pontos obtidos experimentalmente e registrados no diagrama tensão-deformação. 


\section{CENTRO EDUCACIONAL NOSSA SENHORA AUXILIADORA \\ INSTITUTOS SUPERIORES DE ENSINO DO CENSA \\ CENTRO DE PESQUISA E PÓS-GRADUAÇÃO}

\section{CONSIDERAÇÕES FINAIS}

A partir do Ensaio de Tração realizado no Laboratório de Ensaios destrutivos do ISECENSA e das questões matemáticas envolvidas neste estudo, desenvolveu-se um trabalho interdisciplinar, em que os alunos vivenciaram a aplicação de conteúdos matemáticos estudadas por eles, durante o curso de Engenharia.

Este trabalho foi importante tanto para os professores envolvidos, pois viram seus ensinamentos serem aplicados, quanto para os alunos que utilizaram os conhecimentos estudados por eles de forma integrada. A interação entre professores e alunos proporcionou a troca de experiências, a integração de saberes e motivou o aprendizado.

Durante o desenvolvimento do projeto, observou-se que os alunos tiveram uma experiência positiva, ampliando seus conhecimentos matemáticos e integrando-os com suas realidades profissionais. Os alunos tiveram uma participação ativa, realizando ensaios e efetuando cálculos sempre em grupo.

Dentre as diversas contribuições que a atividade em equipe proporciona, podemos destacar que neste trabalho ela favoreceu a troca de experiências, o desenvolvimento das tarefas, contribuiu para o engajamento dos alunos nas discussões dos conteúdos e proporcionou tomadas de decisão, desenvolvendo habilidades matemáticas e a competência crítica.

Consideramos que esse tipo de trabalho dá significado ao estudo da disciplina de Cálculo nos cursos de Engenharia e faz com que os alunos tenham um olhar diferenciado para o estudo das disciplinas técnicas, percebendo o quanto a Matemática é importante no seu curso de Engenharia e que esta fará a diferença quando estiverem em suas atividades profissionais como Engenheiros.

Diante do exposto, a questão de pesquisa foi satisfatoriamente respondida e os objetivos delineados neste trabalho foram alcançados.

\section{REFERÊNCIAS}

BEER, Ferdinand P.; JOHNSTON, E. R.; DEWOLF, John T.; MAZUREK, David F. Mecânca dos Materiais. São Paulo: AMGH Editora, 2015.

CALLISTER JR, William D.; RETHWISCH, David G. Ciência e Engenharia de Materiais: uma introdução. Rio de Janeiro: LTC, 2013.

CAMPOS, Lídio Mauro Lima. Uso de Ferramentas Educacionais na Disciplina de Cálculo Diferencial e Integral. Artigo. Disponível em <http://tecnologiasnaeducacao.pro.br/wp-content/uploads/2015/07/Art-13vol1-dez-20091.pdf>. Acesso em 31/07/2015.

HIBBELER, Russell Charles. Resistência dos materiais. São Paulo: Pearson Prentice Hall, 2010.

LOPES, Jorge Teófilo de Barros. Estrutura e propriedades dos materiais. Apostila. Disponível em: $<$ http://jorgeteofilo.files.wordpress.com/2011/08/epm-apostila-capc3adtulo09-propriedades.pdf $>$. Acesso em $\underline{12 / 10 / 2013}$.

TOMAZ, Vanessa Sena; DAVID, Maria Mauela Martins Soares. Interdisciplinaridade e aprendizagem da matemática em sala de aula. Coleção Tendências em Educação Matemática.3.ed. Belo Horizonte: Autêntica Editora, 2013. 